\title{
A Flexible Bound Admission Control Algorithm for Vertical Handover in Ubiquitous Environment
}

\author{
Jong Min Lee ${ }^{1}$, Ok Sik Yang ${ }^{1}$, Seong Gon Choi ${ }^{2}$, and Jun Kyun Choi ${ }^{1}$ \\ ${ }^{1}$ Information and Communications University (ICU), \\ 119 Munji-Dong, Yuseong-Gu, Daejeon 305-732, Republic of Korea \\ \{jmlee, yos, jkchoi\}@icu.ac.kr \\ ${ }^{2}$ Chungbuk National University (CBNU), \\ 12 Gaeshin-Dong, Heungduk-Gu, Chungbuk 361-763, Republic of Korea \\ sgchoi@chungbuk.ac.kr
}

\begin{abstract}
In this paper, we present FBAC (Flexible Bound Admission Control) algorithm using softness profile to reduce handover blocking probability over WLAN and WAAN (Wide Area Access Network). FBAC algorithm utilizes dynamic resource allocation scheme to decrease the blocking probability of vertical handover connections within the limited capacity of system. Based on FBAC algorithm, we derive the handover blocking probability as new traffic load and handover traffic load increase. In order to evaluate the performance, we compare FBAC algorithm against traditional non-bounded and fixed bound schemes. Numerical results show that the FBAC scheme improves handover blocking probability in ubiquitous environment.
\end{abstract}

\section{Introduction}

Efficient resource management for multimedia applications with different quality of service (QoS) requirements in the presence of heterogeneous wireless access technologies (e.g. 3G cellular, IEEE 802.11 WLAN, Bluetooth) is one of the most challenging issues for ubiquitous environment. In such environment, a users or network will be able to decide where to handover among the different access technologies based on the bandwidth, cost, and user preferences, application requirements and so on. Therefore, efficient radio resource management and connection admission control (CAC) strategies will be key components in such a heterogeneous wireless system supporting multiple types of applications with different QoS requirements [1].

Many admission control schemes have been proposed to enable the network to provide the desired QoS requirements by limiting the number of admitted connections to that network to reduce or avoid connection dropping and blocking [2], [3]. In ubiquitous environment, other aspects of admission control need to be considered due to handover. If the wireless network is unable to assign a new channel due to the lack of resources, an accepted connection may be dropped before it is terminated as a result of the mobile user moving from its current place to another during handover. Since dropping an ongoing connection is generally more sensitive to a mobile user than blocking a new connection request, handover connections should have a higher 
priority over the new connections in order to minimize the handover blocking probability. On the other hand, reducing the blocking of handover connection by channel reservation or other means could increase blocking for new connections. There is therefore a trade off between these two QoS measures [4]. The problem of maintaining the service continuity and QoS guarantees to the multimedia applications during handover is deteriorated by the increase of vertical handover in heterogeneous wireless networks.

In the ubiquitous environment, vertical handover considering user preferences, traffic characteristic, user mobility range, and so on could occur more frequently than horizontal handover. Therefore, vertical handover should have higher priority to support QoS requirement because it considers more various factors (e.g. cost, bandwidth, velocity, etc.) than horizontal handover. So we proposed a dynamic admission control for vertical handover connections in ubiquitous environment.

This paper is organized as follows. In the next section, we describe the architecture of proposed algorithm. In section 3, we propose a flexible admission control algorithm using softness profile. Numerical results obtained using the traditional methods are presented and compared in Section 4. Finally, we conclude the paper in section 5.

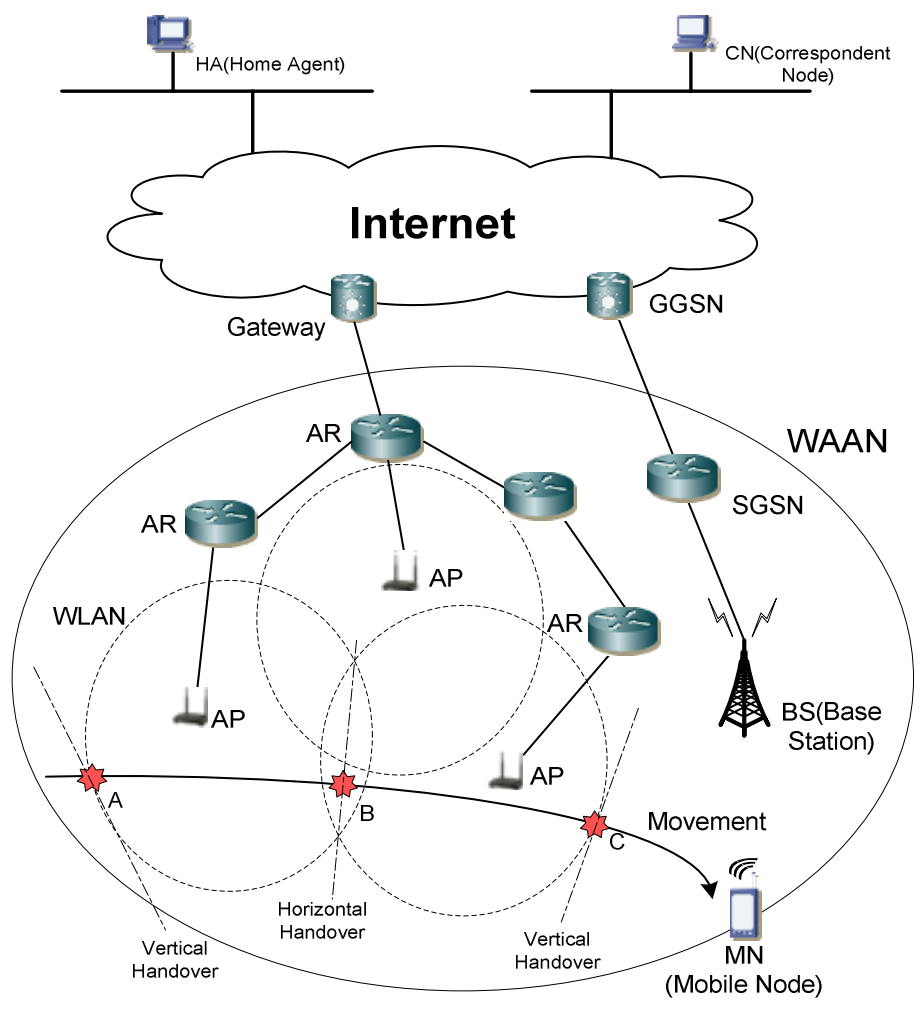

Fig. 1. Network architecture for mobility service in ubiquitous environment 


\section{The Architecture of Proposed Algorithm}

\subsection{Network Architecture}

Fig. 1 shows the network architecture for mobility service in ubiquitous environment. This architecture is based on IPv6 networks to support the movement of every user. There are two kinds of users that can handover under this architecture. One is WLAN to WAAN users and vice versa. Therefore, there will be two handovers between WLAN and WAAN: vertical handover and horizontal handover.

We assume that a user has multi-interface terminal [5]. As shown in Fig. 1, the connection initiated in WAAN and mobile node is moving to right side. When the mobile node jumps into another access area, it requires vertical or horizontal handover connection to continue their movement or connection, where $\mathrm{A}, \mathrm{B}$ and $\mathrm{C}$ are handover points respectively. A loosely coupled inter-working approach can be considered for implementation. In this case, Mobile IP [6] mechanism must be implemented in all equipment including MNs. This approach also should support more than one IP address for one mobile user so that one user can access more than one wireless system simultaneously. Finally, on the top of a network, a suitable resource allocation mechanism is required to control the traffic and system load. FBAC algorithm is exploited here in this architecture [7].

\section{Proposed FBAC Algorithm}

\subsection{Softness Profile}

As shown in Fig. 2, the softness profile is defined on the scales of two parameters: satisfaction index and bandwidth ratio [8]. The satisfaction index is a mean-opinionbased (MOS) value graded from 1 to 5, which is divided by two regions: the acceptable satisfaction region and low satisfaction region.

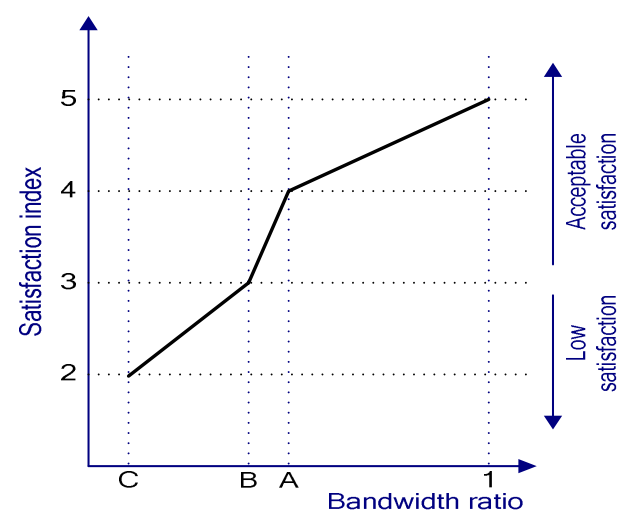

Fig. 2. Softness profile 
Bandwidth ratio graded from 0 to 1 can be separated by 3 regions. In the region from 1 to A, it has low degradation of satisfaction index. It means users are not sensitive in this region. However, it has large degradation of satisfaction index in the region from A to B.

The point indicated as B is called the critical bandwidth ratio $(\xi)$ used in proposed algorithm. Since this value is the minimum acceptable satisfaction index, it can be threshold of bandwidth ratio. In the region from B to 0 , users do not satisfy their services. Therefore, this region should not be assigned to any users. Generally, the critical bandwidth ratio $(\xi)$ of Video On Demand (VOD) is $0.6 \sim 0.8$ and back ground traffic is $0.2 \sim 0.6$ [9].

\subsection{Proposed FBAC Algorithm}

Proposed FBAC algorithm is illustrated in Fig. 3. This algorithm shows dynamic bound handover procedure within given total bandwidth $B_{\text {total }}$. When a mobile node requires bandwidth for new or handover connections, mobile agent checks the available bandwidth within some threshold to decide connection admission or rejection. In

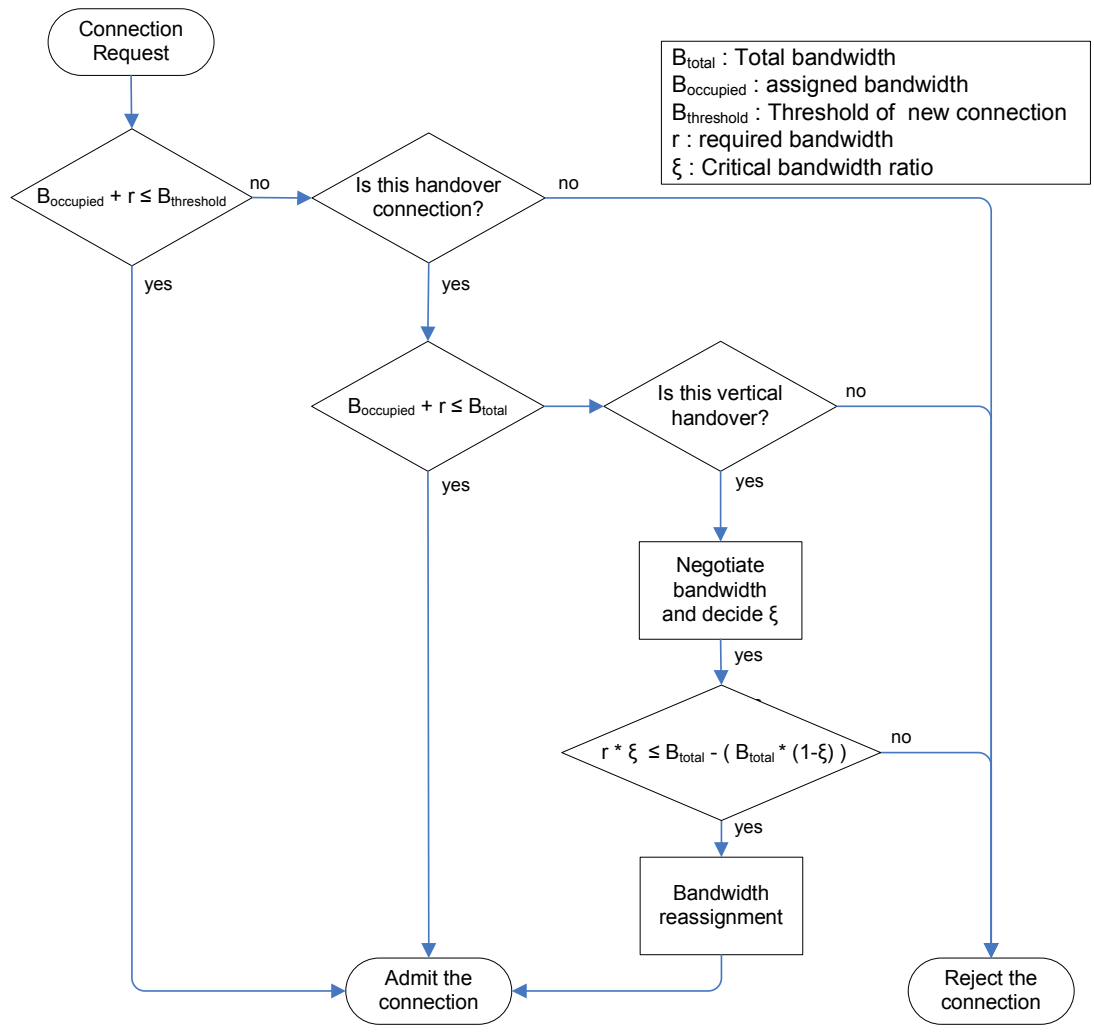

Fig. 3. Proposed FBAC algorithm 


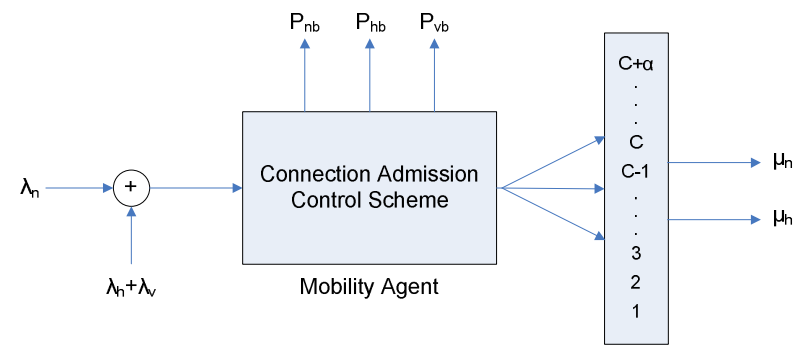

Fig. 4. System model

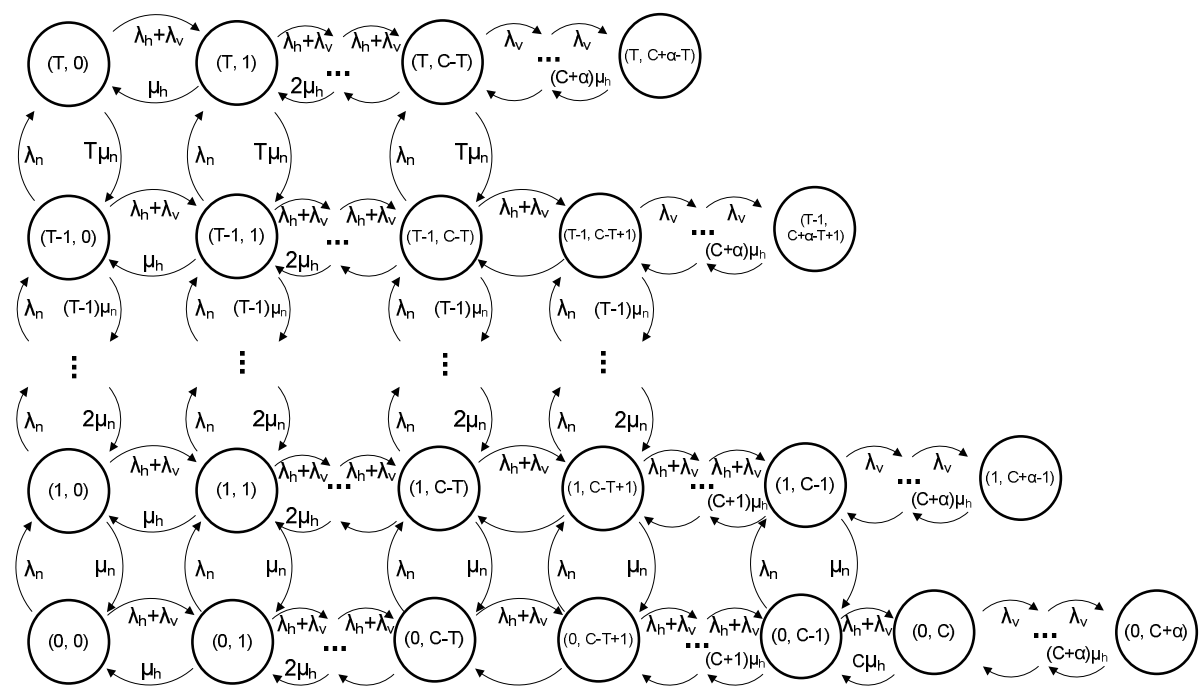

Fig. 5. Transition diagram for the proposed FBAC algorithm

this point, handover connections should be treated differently in terms of resource allocation. Since users tend to be much more sensitive to connection dropping (e.g. disconnection during VOD service) than to connection blocking (e.g. fail to initiate connection), handover connections should assign higher priority than the new connection. Especially, the vertical handover connection needs to have higher priority than horizontal handover connection because it considers more various factors (e.g. cost, bandwidth, velocity, etc.) than horizontal handover connection. In this time, if there is no available bandwidth to accept vertical handover connection, mobile agent negotiates and reassigns bandwidth by choosing critical bandwidth ratio $(\xi)$. As a result, the vertical handover connections can be accepted more than horizontal handover connections.

Fig. 4 shows the system model of the proposed approach. Let $\mathrm{C}$ denote the total capacity and $P_{n b}, P_{h b}, P_{v b}$ denote the blocking probabilities of new connection, horizontal handover and vertical handover connection respectively. The arrival proc- 
ess of new and handover connections is assumed to be Poisson and denoted by $\lambda_{n}$ and $\lambda_{h}+\lambda_{v}$. The factor $\alpha=\lfloor(1-\xi) * C\rfloor$ is bandwidth donated from each user within the same coverage without critical degradation of service. From the assumption, we obtain the blocking probability of horizontal handover connection, vertical handover connection, and new connection.

Fig. 5 indicates the transition diagram for the proposed scheme. The detailed notations used in this diagram are shown in Table. 1.

Table 1. Notations

\begin{tabular}{ll}
\hline Notation & explanation \\
\hline$\lambda_{n}$ & Arrival rate of new connection \\
$\lambda_{h}$ & Arrival rate of horizontal handover connection \\
$\lambda_{v}$ & Arrival rate of vertical handover connection \\
$1 / \mu_{h}$ & Average channel holding time for handover connections \\
$1 / \mu_{n}$ & Average channel holding time for new connections \\
$C$ & Maximum number of server capacity \\
$T$ & Threshold (bound of the total bandwidth of all accepted \\
$\xi$ & new connections) \\
$\alpha$ & Critical bandwidth ratio \\
$n_{n}$ & $\lfloor(1-\xi) * C\rfloor$ \\
$n_{h v}$ & Number of new connections initiated in the coverage \\
\hline
\end{tabular}

In order to analyze the blocking probability of each connection, we use the twodimensional Markov chain model with the state space $\mathrm{S}$ and $\mathrm{M} / \mathrm{M} / \mathrm{C}+\alpha / \mathrm{C}+\alpha[10]$ model is utilized.

$$
S=\left\{\left(n_{n}, n_{h v}\right) \mid 0 \leq n_{n} \leq T,\left(n_{n}+n_{h v}\right) \leq C+\alpha\right\}
$$

Let $q\left(n_{n}, n_{h v} ; \overline{n_{n}}, \overline{n_{h v}}\right)$ denote the probability transition rate from state $\left(n_{n}, n_{h v}\right)$ to $\left(\overline{n_{n}}, \overline{n_{h v}}\right)$. Then, we obtain the below.

$$
\begin{array}{ll}
q\left(n_{n}, n_{h v} ; n_{n}+1, n_{h v}\right)=\lambda_{n} & \left(0 \leq n_{n}<T, 0 \leq n_{h v} \leq C\right) \\
q\left(n_{n}, n_{h v} ; n_{n}-1, n_{h v}\right)=n_{n} \mu_{n} & \left(0<n_{n} \leq T, 0 \leq n_{h v} \leq C\right) \\
q\left(n_{n}, n_{h v} ; n_{n}, n_{h v}+1\right)=\lambda_{h}+\lambda_{v} & \left(0 \leq n_{n} \leq T, 0 \leq n_{h v}<C\right) \\
q\left(n_{n}, n_{h v} ; n_{n}, n_{h v}-1\right)=n_{h v} \mu_{h} & \left(0 \leq n_{n} \leq T, 0<n_{h v} \leq C\right) \\
q\left(n_{n}, n_{h v} ; n_{n}, n_{h v}+1\right)=\lambda_{v} & \left(0 \leq n_{n} \leq T, C \leq n_{h v}<C+\alpha\right) \\
q\left(n_{n}, n_{h v} ; n_{n}, n_{h v}-1\right)=n_{h v} \mu_{h} & \left(0 \leq n_{n} \leq T, C<n_{h v} \leq C+\alpha\right)
\end{array}
$$


Let $p\left(n_{n}, n_{h v}\right)$ denote the steady-state probability that there are new connections $\left(n_{n}\right)$ and vertical and horizontal handover connections $\left(n_{h v}\right)$. By using the local balance equation [10], we can obtain

$$
\begin{aligned}
& p\left(n_{n}, n_{h v}\right)=\frac{\rho_{n}{ }^{n} \cdot \rho_{h}{ }^{h v} \cdot p(0,0)}{n_{n} ! n_{h v} !} \\
& \text { where }\left(0 \leq n_{n} \leq T, 0 \leq n_{h v}<C\right), \rho_{n}=\frac{\lambda_{n}}{\mu_{n}}, \rho_{h}=\frac{\lambda_{h}+\lambda_{v}}{\mu_{h}} \\
& p\left(n_{n}, n_{h v}\right)=\frac{\rho_{n}{ }^{n} \cdot \rho_{h}^{C} \cdot \rho^{h v-C} p(0,0)}{n_{n} ! n_{h v} !} \\
& \text { where }\left(0 \leq n_{n} \leq T, C \leq n_{h v} \leq C+\alpha\right), \rho=\frac{\lambda_{n}}{\mu_{n}}, \rho=\frac{\lambda_{v}}{\mu_{h}}
\end{aligned}
$$

From the normalization equation, we also obtain

$$
\begin{aligned}
& p(0,0)=\left[\sum_{0 \leq n_{n} \leq T, n_{n}+n_{h v} \leq C+\alpha} \frac{\rho_{n}^{n_{n}}}{n_{n} !} \cdot \frac{\rho_{h}^{n_{h v}}}{n_{h v} !}\right]^{-1} \\
& =\left[\sum_{n_{n}=0}^{T} \frac{\rho_{n}{ }^{n_{n}}}{n_{n} !} h \cdot \sum_{n_{h v}=0}^{C-n_{n}} \frac{\rho_{h}{ }^{n}{ }_{h v}}{n_{h v} !}+\sum_{n_{n}=0}^{T} \frac{\rho_{n}{ }^{n_{n}}}{n_{n} !} \cdot \sum_{n_{h v}=C}^{(C+\alpha)-n_{n}} \frac{\rho_{h}{ }^{n_{h v}} \cdot \rho_{h}{ }^{\left(n_{h v}-C\right)}}{n_{h v} !}\right]^{-1}
\end{aligned}
$$

From this, we obtain the formulas for new connection blocking probability and handover connection blocking probability as follows:

$$
\begin{aligned}
& P_{n b}=\frac{\sum_{n_{h v}=0}^{C-T} \frac{\rho^{T}}{T !} \cdot \frac{\rho^{n_{h v}}}{n_{h v} !}+\sum_{n_{n}=0}^{T-1} \frac{\rho^{n_{n}}}{n_{n} !} \cdot \frac{\rho_{h}{ }^{C-n_{n}}}{\left(C-n_{n}\right) !}}{P(0,0)} \\
& P_{h b}=\frac{\sum_{n_{n}=0}^{T} \frac{\rho^{n_{n}}}{n_{n} !} \cdot \frac{\rho_{h}{ }^{C-n_{n}}}{\left(C-n_{n}\right) !}}{P(0,0)} \\
& P_{v b}=\frac{\sum_{n_{n}=0}^{T} \frac{\rho^{n_{n}}}{n_{n} !} \cdot \frac{\rho_{h}{ }^{(C+\alpha)-n_{n}}}{\left((C+\alpha)-n_{n}\right) !}}{P(0,0)}
\end{aligned}
$$

Obviously, when $\mathrm{T}$ is equal to $\mathrm{C}$, the new connection bounding scheme becomes the non-prioritized scheme. As we expect, we can obtain [3]

$$
P_{n b}=P_{h b}=P_{v b}=\frac{\frac{\left(\rho_{n} \rho_{h}\right)^{C}}{C !}}{\sum_{n=0}^{C} \frac{\left(\rho_{n} \rho_{h}\right)^{n}}{n !}}
$$




\section{Numerical Results}

In this section, we present the numerical results for the comparison of performance. We compared three bounding schemes: non-bound, fixed bound, and FBAC algorithms. Fig. 6 shows handover blocking probability under the following parameters: $\mathrm{C}=30, \mathrm{~T}=15, \lambda_{n}=1 / 30, \lambda_{h}=1 / 60, \lambda_{v}=1 / 60, \mu_{h}=1 / 450, \mu_{n}$ is varying from $1 / 600$ to $1 / 200$, and $\xi=0.9$.

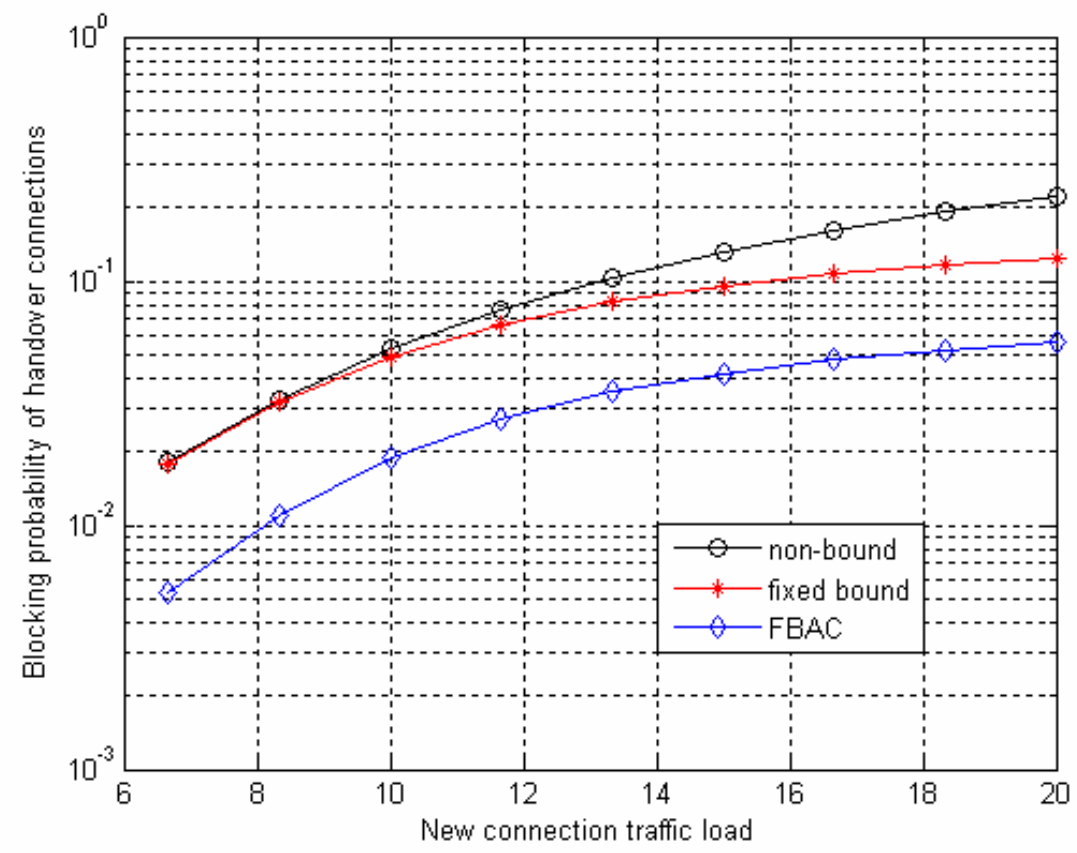

Fig. 6. Blocking probability of handover connections vs. new connection load

In Fig. 6 handover connection traffic load is given as $\rho_{h}=15$. It is observed that when traffic load of the handover connection is higher than the new connection traffic load (e.g. $\rho_{h}>\rho$ ), non-bound scheme and fixed bound scheme are not much different. On the other hand, when traffic load of the handover connection is lower than the new connection traffic (e.g. $\rho_{h}<\rho$ ), their blocking probabilities become different. This is the case when the new connections arrive in bursts (say after finishing a class) [11]. Our proposed FBAC algorithm can offer the seamless handover for ongoing connections with lower blocking probability.

In Fig. 7, we increase the handover connection traffic load $\left(\rho_{h}\right)$. This graph shows the blocking probability of handover connection under the following parameters: 
$\mathrm{C}=30, \mathrm{~T}=20, \lambda_{n}=1 / 20, \lambda_{h}=1 / 60, \lambda_{v}=1 / 60, \mu_{n}=1 / 300, \mu_{h}$ is varying from $1 / 100$ to $1 / 1200$, and $\xi=0.9$.

In this case, traffic load of handover connections $\left(\rho_{h}\right)$ are increasing from 0 to 40 and traffic load $\left(\rho_{n}\right)$ of the new connection is 15 . Since handover connections are not bounded, as increasing the handover traffic load, the differences among three schemes become similar.

In summary, the FBAC algorithm could achieve better results than the traditional bounding schemes, especially for the handover connection. These results can be used for analysis and design of ubiquitous environment.

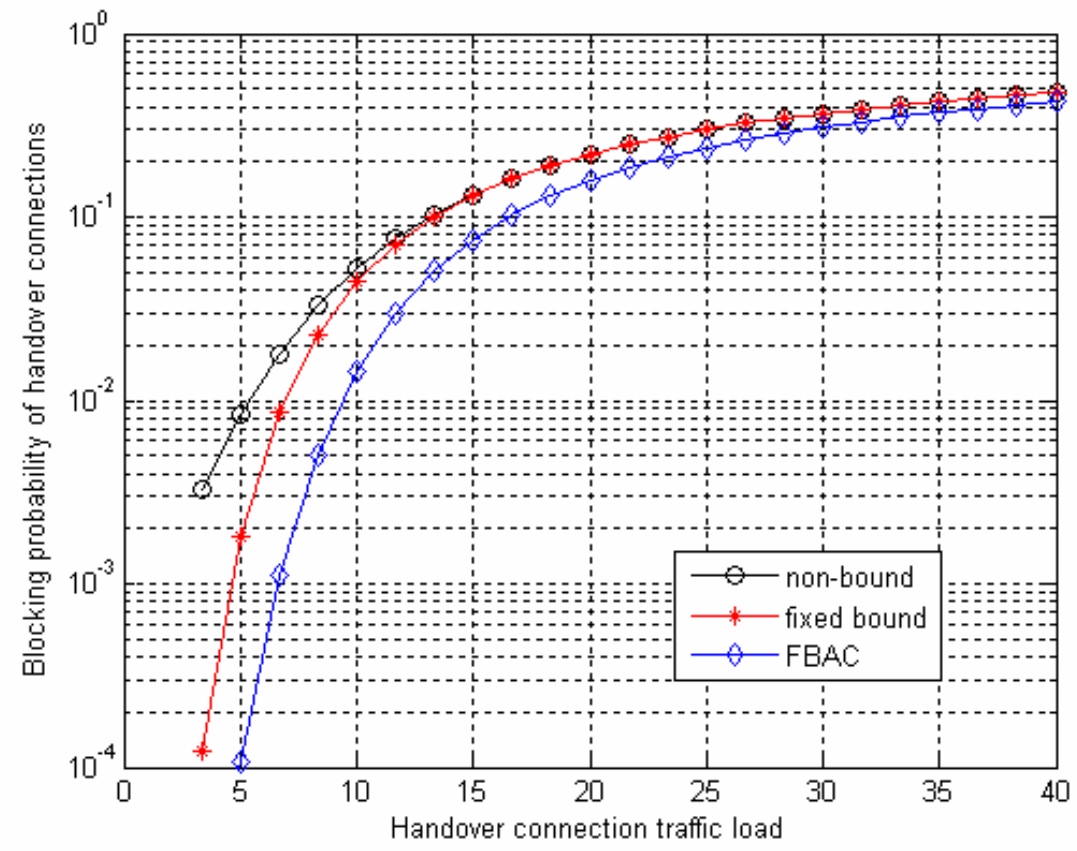

Fig. 7. Blocking probability of handover connections vs. handover connection load

\section{Conclusion}

In this paper, we proposed FBAC algorithm that reduces the blocking probability of vertical handover connections within the limited capacity of system over ubiquitous environment (e.g. WLAN and WAAN). Proposed FBAC algorithm considers vertical handover connections that have higher priority. In order to analyze the blocking probability of FBAC algorithm, we use the two-dimensional Markov chain model. From the numerical analysis, we compared FBAC algorithm against traditional non-bound and fixed bound scheme. As a result, proposed FBAC scheme is able to improve 
handover blocking probability in ubiquitous environment. Future work needs to analyze the optimized critical bandwidth ratio based on the number of user arrival.

Acknowledgement. This work was supported in part by the MIC, Korea under the ITRC program supervised by the IITA and the KOSEF under the ERC program.

\section{References}

1. Dusit Niyato, Ekram Hossain: Call Admission Control for QoS Provisioning in 4G Wireless Networks: Issues and Approaches, IEEE Network, Sept.-Oct. (2005) 5-11

2. I. Katzela, M. Naghshineh: Channel assignment schemes for cellular mobile telecommunication systems: A comprehensive survey, IEEE Personal Commun., vol. 3, June (1996) 10-31

3. Yuguang Fang, Yi Zhang: Call Admission Control Schemes and Performance Analysis in Wireless Mobile Networks, IEEE Transactions on Vehicular Technology, Vol. 51, No. 2, March (2002) 371-382

4. Emre A. Yavuz, Victor C. M. Leung: A Practical Method for Estimating Performance Metrics of Call Admission Control Schemes in Wireless Mobile Networks, IEEE WCNC, March (2005) 1254-1259

5. M. Buddhikot et al.: Design and Implementation of a WLAN/CDMA2000 Interworking Architecture, IEEE Communications Magazine, Nov. (2003)

6. D. Johnson, C. Perkins and J. Arkko,: Mobility Support for IPv6, RFC 3775, June (2004)

7. Sheng-Tzong Cheng, Jian-Liang Lin: IPv6-Based Dynamic Coordinated Call Admission Control Mechanism Over Integrated Wireless Networks, IEEE Journal on Selected Areas in Communications, Vol. 23, No. 11, Nov. (2005) 2093-2103

8. Reininger D., Izmailov R.: Soft quality-of-service control for multimedia traffic on ATM networks, Proceedings of IEEE ATM Workshop, (1998) 234-241

9. Sung H. Kim, Yeong M. Jang: Soft QoS-Based Vertical Handover Scheme for WLAN and WCDMA Networks Using Dynamic Programming Approach, LNCS 2524, Nov. (2002) 707-716

10. Kleinrock. L.: Queueing System, Vol. 1 Theory, John Wiley and Sons, New York (1975)

11. Jiongkuan Hou, Yuguang Fang: Mobility-based call admission control schemes for wireless mobile networks, Wireless Communications and Mobile Computing. Wirel. Commun. Mob. Comput. (2001) 1:269-282 